\title{
Low-Cost Optoelectronic Self-Injection-Locked Oscillators
}

\author{
Kwang-Hyun Lee, Jae-Young Kim, Woo-Young Choi, Hideki Kamitsuna, Minoru Ida, and Kenji Kurishima
}

\begin{abstract}
We demonstrate a new configuration for an optoelectronic self-injection-locked (SIL) oscillator, where a part of the electrical output signal is self-injected after passing through a long optical delay line for output phase-noise reduction. The SIL oscillator consists of an electrical free-running oscillator and a long optical feedback loop. For the compact and low cost configuration, the free-running oscillator is realized with an InP HPT-based monolithic oscillator and electrical-to-optical conversion is carried out by two low-speed and low-cost laser diodes. With this new configuration, we achieve more than 55-dB phase-noise reduction at $10-\mathrm{kHz}$ frequency offset from the center frequency of about $10.8 \mathrm{GHz}$ by injecting $8-\mathrm{dBm}$ optical signals without using any high-speed optoelectronic components.
\end{abstract}

Index Terms-InP monolithic oscillator, optoelectronic oscillator (OEO), phase-noise reduction, self-injection locking (SIL).

\section{INTRODUCTION}

CELF-INJECTION-LOCKED (SIL) oscillators can generate stable and low-phase noise signals by injecting a part of output signals into the oscillator through a high-quality $(Q)$-factor external resonator, a delay line, or an amplifier [1], [2]. The higher $Q$ external resonator or longer delay line can produce lower output phase noises for the fixed self-injection power. However, it is not an easy task to realize a long delay line with RF cables due to their large loss and high- $Q$ devices are not easily available especially at very high frequencies [3].

In order to solve the above problems, an optoelectronic SIL oscillator composed of an electrical free-running oscillator and an optical feedback route has been proposed and demonstrated [4]-[6]. In this oscillator, optical fiber having the length of a few kilometers is used for the feedback route with its low transmission loss and flexible feature. With this long optical delay line, the optoelectronic SIL oscillator can generate low-phase noise signals. Moreover, it can produce optical signals modulated by the resulting low phase-noise $\mathrm{RF}$ signals, which are useful for photonic applications such as radio-on-fiber systems [6]. As a compact realization method, utilization of heterojunction phototransistor (HPT) for electrical free-running oscillator was reported [7]. In this method,

Manuscript received December 3, 2007; revised April 10, 2008. The work of K.-H. Lee, J.-Y. Kim, and W.-Y. Choi was supported by the Basic Research Program of the Korea Science and Engineering Foundation.

K.-H. Lee, J.-Y. Kim, and W.-Y. Choi are with the Department of Electrical and Electronic Engineering, Yonsei University, Seoul 120-749, Korea (e-mail: wchoi@yonsei.ac.kr).

H. Kamitsuna, M. Ida, and K. Kurishima are with the NTT Photonics Laboratories, NTT Corporation, Kanagawa 243-0198, Japan (e-mail: Kamituna @ aecl. ntt.co.jp).

Color versions of the figures in this letter are available online at http://ieeexplore.ieee.org.

Digital Object Identifier 10.1109/LPT.2008.925189

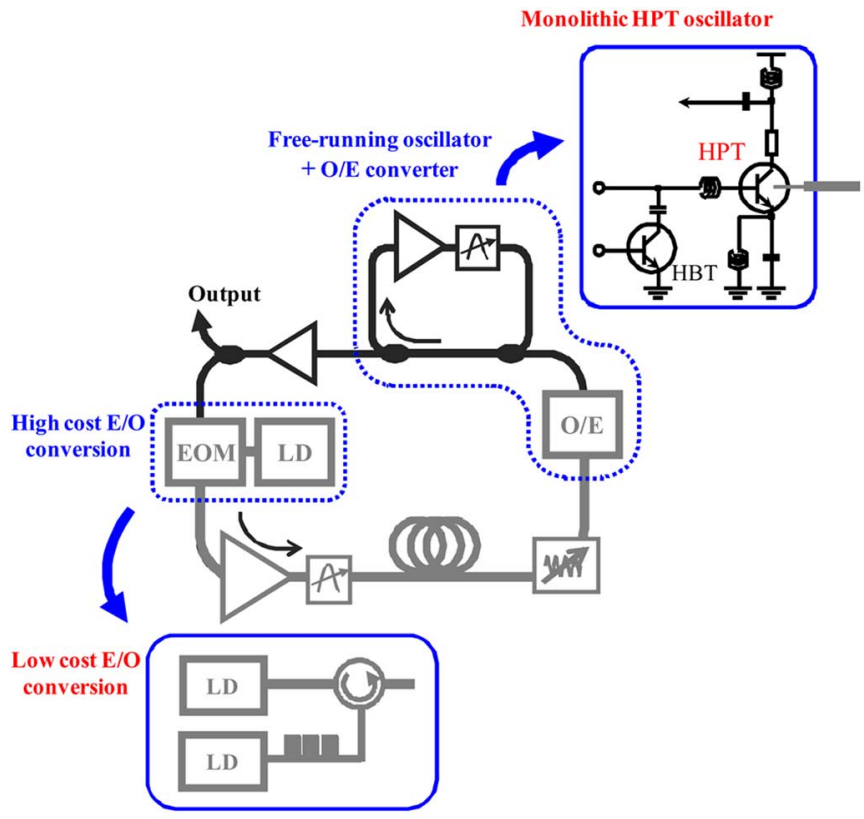

Fig. 1. Compact and low cost configuration for an optoelectronic SIL oscillator based on a monolithic HPT oscillator and low cost optical components.

the HPT also performs optical-to-electrical (O/E) conversion at the end of optical feedback loop.

In this letter, a simpler and more cost-effective configuration for optoelectronic SIL oscillator is proposed and demonstrated. In our scheme, shown in Fig. 1, the electrical free-running oscillator and $\mathrm{O} / \mathrm{E}$ converter is realized with a monolithic HPT oscillator [8]. Moreover, electrical-to-optical (E/O) conversion is performed with low-speed distributed feedback (DFB) and Fabry-Pérot (FP) laser diodes (LDs). This E/O conversion scheme is essentially the same as the technique we previously reported for overcoming LD modulation bandwidth limitation [9]. Note that no high-speed high-cost optical/optoelectronic components such as external modulators or mode-locked lasers are used in our scheme. More than 55-dB phase-noise reduction at $10-\mathrm{kHz}$ frequency offset from the center frequency of $10.8-\mathrm{GHz}$ is achieved with $8-\mathrm{dBm}$ optical signal injection.

\section{OPERATING PRINCIPLE}

As can be seen in Fig. 1, a part of output signals from the HPT monolithic oscillator is injected to an E/O converter and converted to optical signals. The converted optical signals are injected into the HPT oscillator after passing through a long optical delay line, achieving self-injection locking for output phase-noise reduction. 


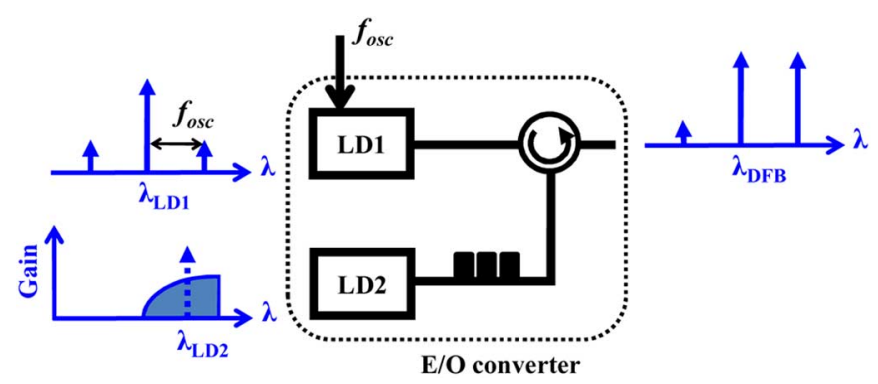

Fig. 2. E/O converter and schematic optical spectra. The shade region means the gain wavelength range of $\mathrm{LD}_{2}$ and $\lambda \mathrm{LD}_{2}$ is the wavelength of $\mathrm{LD} 2$ without any optical injection.

The E/O converter is configured with two LDs, one of which is a commercial 2.5-Gb/s DFB LD (LD1) and the other is a commercial FP LD (LD2) without an isolator. As shown in Fig. 2, LD1 is directly modulated by injected RF signals, producing two side bands in the wavelength domain. These side bands are very weak since LD1 is not fast enough for injected RF signals. Once these optical modes are injected into LD2 through an optical circulator, only the optical modes within the gain wavelength range of LD2 obtain optical gain. The gain wavelength range marked by the shade region in Fig. 2 is determined by LD2 lasing wavelength and the injected optical power [9]. By controlling the temperature or the bias current for LD2, only the desired side mode can be amplified and this results in enhancement of the modulation depth as well as the single sideband optical spectrum, which can avoid the RF signal-fading problem induced by fiber dispersion [10].

The O/E conversion is performed by the HPT oscillator. The $\mathrm{In}_{0.53} \mathrm{Ga}_{0.47}$ As layer of the HPT detects $1.55-\mu \mathrm{m}$ optical signals and the photodetected signals lock the HPT free-running oscillator, inducing self-injection locking. Once the oscillator is self-locked by the delayed replica of its output signal, its frequency and phase fluctuations are reduced [4]. The phase-noise reduction ratio $(\eta)$ near the carrier frequency can be described as [4]

$$
\lim _{\omega \rightarrow 0} \eta(\omega) \rightarrow \frac{1}{\left(1+\sqrt{\kappa} \omega_{3 \mathrm{~dB}} \tau\right)^{2}}
$$

where $\omega$ is the offset frequency from the center frequency, $\omega_{3}$ $\mathrm{dB}$ is the half-width-at-half-maximum of the resonator spectrum for the HPT oscillator, $\tau$ is the optical-loop delay, and $\kappa$ is the self-injection power normalized to the oscillator output power given as

$$
\kappa=\frac{\left(P_{\mathrm{opt}} \cdot \rho\right)^{2} R}{P_{\mathrm{out}}}
$$

where $P_{\mathrm{opt}}$ is the injected optical power, $\rho$ is the photoresponsivity, and $R$ is the load impedance of the HPT and $P_{\text {out }}$ is the oscillator output power. From these two equations, it becomes clear that a longer delay line or larger optical power injection produces more phase noise reduction.

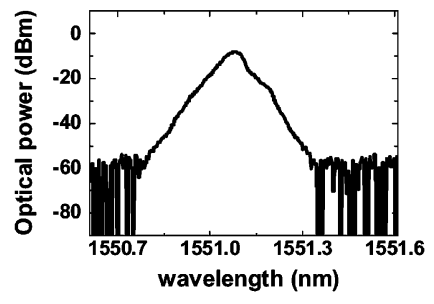

(a)

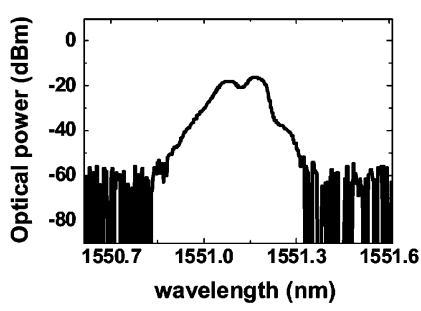

(b)
Fig. 3. Measured optical spectra of the output signals from (a) the directly modulated LD1 by the HPT oscillator output signal and (b) the LD2 with optical injection of output signals from LD1.

\section{EXPERIMENTAL SETUP AND RESUlTS}

The HPT oscillator used in this investigation has the optical window with 5- $\mu \mathrm{m}$ diameter on the top of the emitter for photodetection and the chip size is only $0.7 \times 0.54 \mathrm{~mm}$. The detailed description of the HPT oscillator can be found in [8]. The free-running frequency and quality factor of the oscillator can be controlled by adjusting the bias voltage for the HBT inserted in the oscillator. In this investigation, the bias was set for $10.8-\mathrm{GHz}$ oscillation.

A total of $50 \%$ of the output signal from the HPT oscillator directly modulated the DFB laser and the modulated optical signal was injected into the FP-LD. The optical spectra measured before and after the FP-LD are shown in Fig. 3(a) and (b), respectively. Although the resolution bandwidth $(0.07 \mathrm{~nm})$ of the used optical spectrum analyzer (OSA) was not high enough to distinguish each mode, Fig. 3(b) clearly shows that the side mode located at the longer wavelength was amplified by the FP-LD.

The output signals from the E/O converter passed through a 2.4-km long single-mode fiber and then they were injected into the HPT oscillator. An erbium-doped fiber amplifier (EDFA) and an electrical amplifier having $30-\mathrm{dB}$ gain were used to partially compensate the conversion (E/O/E) loss, and an optical filter was inserted for filtering out EDFA ASE noises.

A total of 50\% of the output signal from the optoelectronic SIL oscillator was measured by an RF spectrum analyzer (HP8563E) and Fig. 4 shows the output spectra measured at different optical power values injected into the HPT oscillator. Fig. 4(a) shows the output spectrum of the free-running HPT oscillator (no optical signal injection), while (b) and (c) are the spectra measured at the injection optical power of -6 and $8 \mathrm{dBm}$, respectively. These figures show that SIL enhances the signal quality and larger injection optical power provides larger improvement. However, unwanted side modes separated by about $84 \mathrm{kHz}$ from the center frequency can be observed in Fig. 4(b) and 4(c). These are due to coupled-loop oscillation [4] and they can be suppressed by adding an additional optical delay line having different length [5].

Fig. 5 shows dependency of single-sideband (SSB) phase noises measured at $10-\mathrm{kHz}$ frequency offset on the optical injection power. As shown in the figure, SSB phase noises reduce with increasing the injection power and the reduction level agrees well with (1) and (2). The inset shows the single-sideband (SSB) phase noises of the output signals shown in Fig. 4. The measured phase-noise values at $10-\mathrm{kHz}$ frequency offset are $-44.17 \mathrm{dBc} / \mathrm{Hz}$ (free-running), 


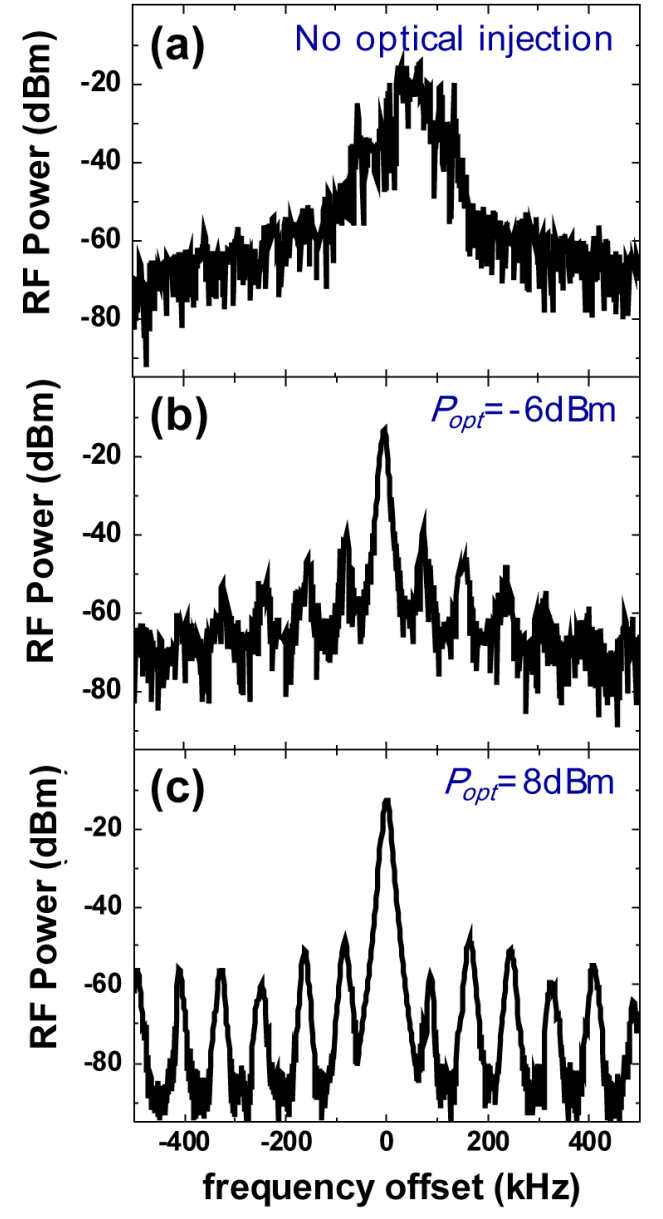

Fig. 4. Measured RF spectra of the output signals for (a) no optical signal injection, (b) $P_{\mathrm{opt}}=-6 \mathrm{dBm}$, and (c) $P_{\mathrm{opt}}=8 \mathrm{dBm}$. The center frequencies are $10.790847,10.790693$, and $10.790314 \mathrm{GHz}$, respectively. In all figures, the frequency span and resolution bandwidth settings were $1 \mathrm{MHz}$ and $10 \mathrm{kHz}, \mathrm{re}-$ spectively.

$-76.83 \mathrm{dBc} / \mathrm{Hz}\left(P_{\mathrm{opt}}=-6 \mathrm{dBm}\right)$, and $-100.00 \mathrm{dBc} / \mathrm{Hz}$ $\left(P_{\text {opt }}=8 \mathrm{dBm}\right)$. Clearly, the self-injection locking drastically enhances the output signal phase quality and about $55-\mathrm{dB}$ phase-noise reduction can be achieved by 8 -dBm optical injection.

\section{CONCLUSION}

We have proposed and demonstrated a compact and low cost optoelectronic SIL oscillator. The proposed scheme does not require any high-speed optical components since $\mathrm{O} / \mathrm{E}$ and $\mathrm{E} / \mathrm{O}$ conversion are performed by an HPT based monolithic oscillator operating as a free-running oscillator and two LDs, respectively. With this scheme, $55-\mathrm{dB}$ phase-noise reduction at $10-\mathrm{kHz}$

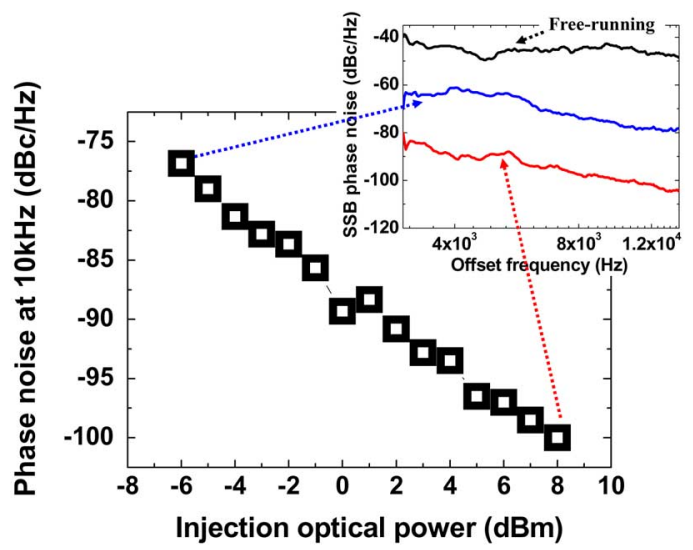

Fig. 5. SSB phase noises at $10-\mathrm{kHz}$ frequency offset from the center frequency versus the injection optical power level. Inset shows the measured phase noises of the optoelectronic output signals at different injection optical power values.

frequency offset from the center frequency of about $10.8 \mathrm{GHz}$ has been successfully demonstrated.

\section{REFERENCES}

[1] H.-C. Chang, "Phase noise in self-injection-locked oscillators," IEEE Trans. Microw. Theory Tech., vol. 51, no. 9, pp. 1994-1999, Sep. 2003.

[2] H.-C. Chang, "Stability analysis of self-injection-locked oscillators," IEEE Trans. Microw. Theory Tech., vol. 51, no. 9, pp. 1989-1993, Sep. 2003.

[3] M. Kaba, H.-W. Li, A. S. Daryoush, J.-P. Vilcot, D. Decoster, J Chazelas, G. Bouwmans, Y. Quiquempois, and F. Deborgies, "Improving thermal stability of opto-electronic oscillators," IEEE Microw. Mag., vol. 7, no. 4, pp. 38-47, Aug. 2006.

[4] K.-H. Lee, J.-Y. Kim, and W.-Y. Choi, "A 30-GHz self-injectionlocked oscillator having a long optical delay line for phase-noise reduction," IEEE Photon. Technol. Lett., vol. 19, no. 24, pp. 1982-1984, Dec. 15, 2007.

[5] D. Eliyahu and L. Maleki, "Low phase noise and spurious level in multi-loop optoelectronic oscillators," in Proc. IEEE Int. Frequency Control Symp., 2003, pp. 405-410.

[6] X. S. Yao and L. Maleki, "Optoelectronic oscillator for photonic systems," IEEE J. Quantum Electron., vol. 32, no. 7, pp. 1141-1149, Jul. 1996.

[7] J. Lasri, A. Bilenca, D. Dahan, V. Sidorov, G. Eisenstein, D. Ritter, and K. Yvind, "A self-starting hybrid optoelectronic oscillator generating ultra low jitter $10-\mathrm{GHz}$ optical pulses and low phase noise electrical signals," IEEE Photon. Technol. Lett., vol. 14, no. 7, pp. 1004-1006, Jul. 2002.

[8] H. Kamitsuna, T. Shibata, K. Kurishima, and M. Ida, "Direct optical injection-locking of InP/InGaAs HPT oscillator ICs for microwave photonics and 40 Gbps-class optoelectronic clock recovery," IEEE Trans. Microw. Theory Tech., vol. 50, no. 12, pp. 3002-3008, Dec. 2002.

[9] H.-S. Ryu, Y.-K. Seo, and W.-Y. Choi, "Dispersion-tolerant transmission of $155-\mathrm{Mb} / \mathrm{s}$ data at $17 \mathrm{GHz}$ using a $2.5-\mathrm{Gb} / \mathrm{s}$-grade DFB laser with wavelength-selective gain from an FP laser diode," IEEE Photon. Technol. Lett., vol. 16, no. 8, pp. 1942-1944, Aug. 2004.

[10] U. Gliese, S. Norskov, and T. N. Nielsen, "Chromatic dispersion in fiber-optic microwave and millimeter-wave links," IEEE Trans. Microw. Theory Tech., vol. 44, no. 10, pp. 1716-1724, Oct. 1996. 